\title{
Direct Sinus Lift and Immediate Loading of Dental Implant- Clinical Reports and Preliminary Radiographic Results
}

\author{
Sharma $\mathrm{S}^{1 *}$, Majumder $\mathrm{K}^{2}$, Din $\mathrm{T}^{3}$, Patel $\mathrm{V}^{4}$ and Shah $\mathrm{M}^{5}$ \\ 1Consultant maxillofacial surgeon, M P Shah Hospital, Nairobi, Kenya \\ ${ }^{2}$ Consultant Orthodontist, M P Shah Hospital, Nairobi, Kenya \\ ${ }^{3}$ Chief Executive Officer (CEO), M P Shah Hospital, Nairobi, Kenya \\ ${ }^{4}$ Medical Director, M P Shah Hospital, Nairobi, Kenya \\ ${ }^{5}$ Chairman, M P Shah Hospital, Nairobi, Kenya
}

Case Report

Volume 4 Issue 1

Received Date: March 16, 2020

Published Date: April 07, 2020

DOI: $10.23880 /$ ijsst-16000143

*Corresponding author: Shalender Sharma, Consultant maxillofacial surgeon, M P Shah Hospital, Nairobi, Kenya, Tel: +254795115136; Email: sharma.shalender@rediffmail.com

\section{Abstract}

Edentulous posterior maxilla is the most challenging area for the implant placement. Because of increase pneumatization of sinus in this area available bone height is inadequate for primary stability of implants. Sinus lift procedure followed by floor augmentation is the only option to increase the availability of bone in this area. Sinus lift procedure can be direct or indirect type depending on available bone height. For augmentation of floor the grafting materials varies from Autogenous bone grafts to xenografts and alloplastic material. We can also use combination of these to increase the success rate. In our cases we used direct sinus lift with MILA SLA Kit and augmentation with Bone Plus TM bone graft with successful immediate implant placement by Anyridge Megagen company in Maxillofacial and Dental Department of M P Shah Hospital, Kenya.

Keywords: Sinus lift; Implants; Bone graft; Ridge augmentation

\section{Introduction}

Edentulism is a condition that keeps many people away from 'feeling their best'. Dental Implants are currently the closest thing technology has to offer in lieu of a natural tooth. Implant success in the posterior maxilla is frequently challenged by unfavorable post-extraction resorptive patterns, pneumatization of the maxillary sinus [1]. The maxillary sinus is the largest of all paranasal sinuses. It lies in the body of the maxilla. It is pyramidal in shape, with its base directed medially towards the lateral wall of the nose, and the apex directed laterally in the zygomatic process of the maxilla [2]. Absence of teeth leads to rapid resorption of alveolar bone in all dimensions due to increased osteoclastic activity in sinus membrane and lack of intraosseous stimulation by periodontal ligament fibers [2]. The outcome of implants depends on the volume and quality of surrounding bone. The presence of lower level of sinus floor in the edentulous area in posterior maxilla limits the implant placement. For gaining the adequate bone height in posterior maxilla we have to do the grafting of the maxillary sinus floor.

The sinus augmentation technique was first discovered by Tatum in 1977 through a lateral window and was first published by Boyne and James in 1980 [3-5]. The original technique of Tatum was later modified by many researchers for the better precision of sinus grafting [6]. After sinus lift for bone grafting there is range of materials available including allografts, xenografts and alloplastic materials. Autogenous bone grafts are always better in outcome than other type of grafts but have other limitations. For the successful outcome of an implant there are primary stability factors and secondary stability factors. Primary factors are implant related and secondary factors are the host environment related [7]. The aim of this case report is to see the possibility and outcome of direct sinus lift with lateral window approach and immediate placement of dental implant. 


\section{Case Reports}

\section{Case Report 1}

A 54-year-old female patient was referred to Department of oral and maxillofacial surgery in M.P Shah Hospital, Kenya for replacement of missing 16. Patient was a known diabetic and she lost her teeth because of periodontal problem. On examination, she was found to have missing 15 and 16. She was being edentulous since last three years. She had been given options for replacement of missing teeth by bridge or implants. Out of that she opted for implants. On radiological examination, (Figure 1) the available bone height was $3 \mathrm{~mm}$ in 16 region which confirmed need for sinus lift procedure.

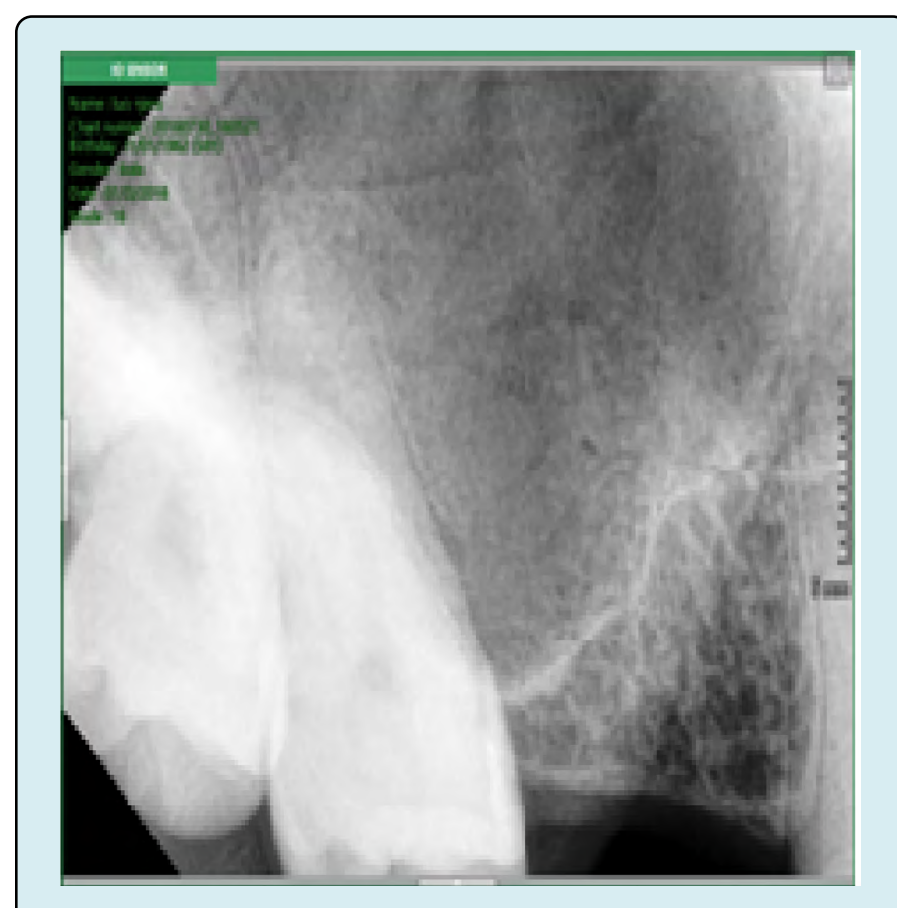

Figure 1: Patient 1 pre-op available bone height.

The same thing explained to the patient and she had given consent for direct sinus lift augmentation and immediate implant placement. Preoperative antibiotic course started 1 day before the operation and continued till $6^{\text {th }}$ post-operative day. A midcrestal, full-thickness incision was performed in the edentulous area along with crevicular incision from 14 till 17. Vertical reliving incision was given along 17 region. Once the flap was reflected, the implant osteotomy site was prepared by carefully preserving the sinus membrane. The cavity was filled using bone graft Bone Plus ${ }^{\mathrm{TM}} 0.5 \mathrm{gm}$ following that Anyridge implant by Megagen size diameter $5.0 \mathrm{~mm}$ and length $11.5 \mathrm{~mm}$ was used. Immediate post-op RVG (Radiovisuograph) \& OPG (Orthopantomogram) was taken (Figure 2). The patient was followed up till two month post-operative day. There was no sign of implant failure. Healing was satisfactory.

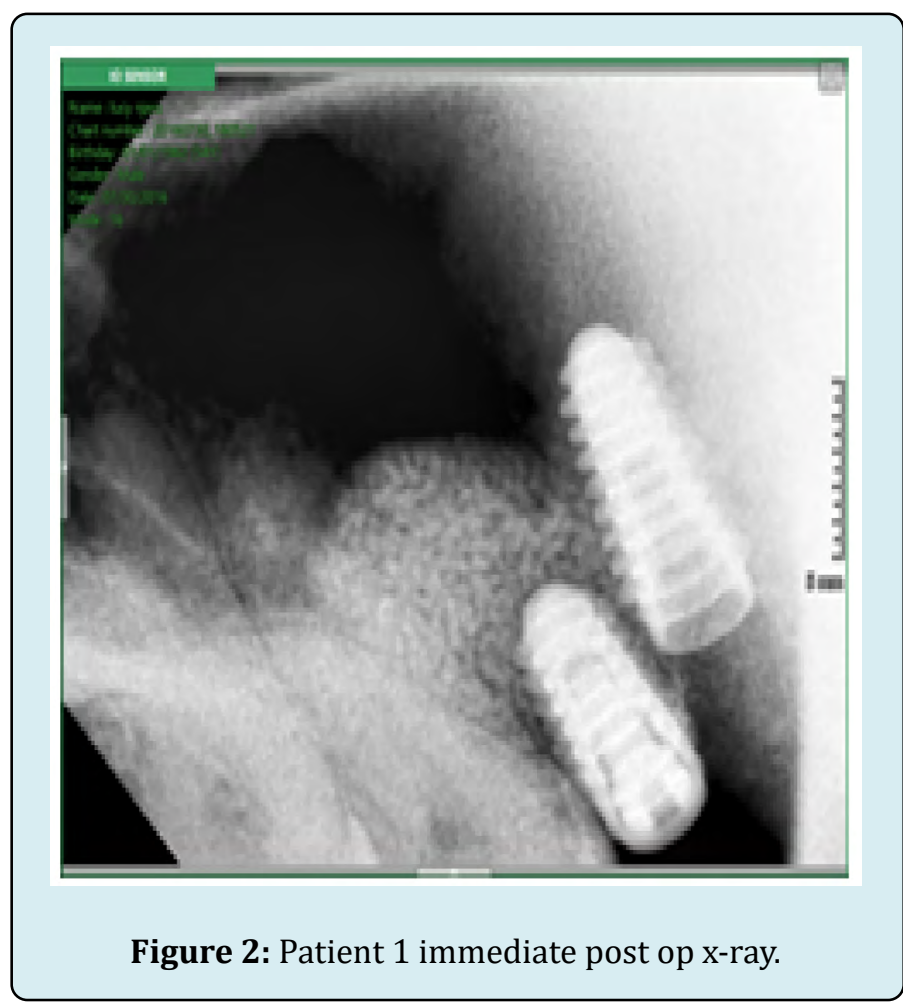

\section{Case Report 2}

A 34-year-old female patient was referred to our Department for replacement of missing 16 with implant. Patient was a known diabetic. On clinical examination missing 15 and 16 was noted. She was edentulous since 1 year. Cause of Edentulism was periapical abscess. She had been given all the options for replacement of missing teeth, out of which she had selected direct sinus lift. On radiological examination, the available bone height in 16 region was $4 \mathrm{~mm}$ which confirmed need for sinus lift procedure (Figure 3). Patient had given consent for direct sinus lift augmentation and immediate implant placement. Preoperative same antibiotic protocol was followed. A midcrestal, full-thickness incision was performed in the edentulous area along with one posterior vertical relieving incision. Once the flap was reflected, the implant osteotomy site was prepared by carefully preserving the sinus membrane. The cavity was filled using bone graft Bone Plus ${ }^{\mathrm{TM}} 0.5 \mathrm{gm}$ following that Anyridge implant by Megagen size diameter $4.0 \mathrm{~mm}$ and length $13 \mathrm{~mm}$ was used. Immediate post-op RVG \& OPG was taken (Figure 4). The patient was followed up till two month post-operative day. There was no sign of implant failure. Healing was satisfactory (Table 1). 


\section{International Journal of Surgery \& Surgical Techniques}

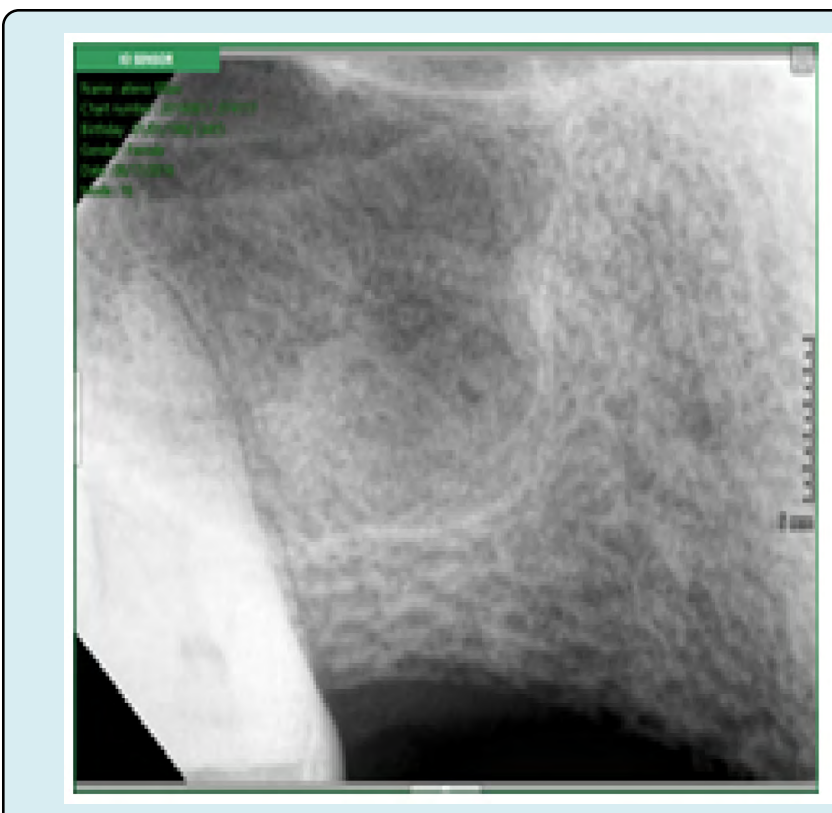

Figure 3: Patient 2 pre-op available bone height.

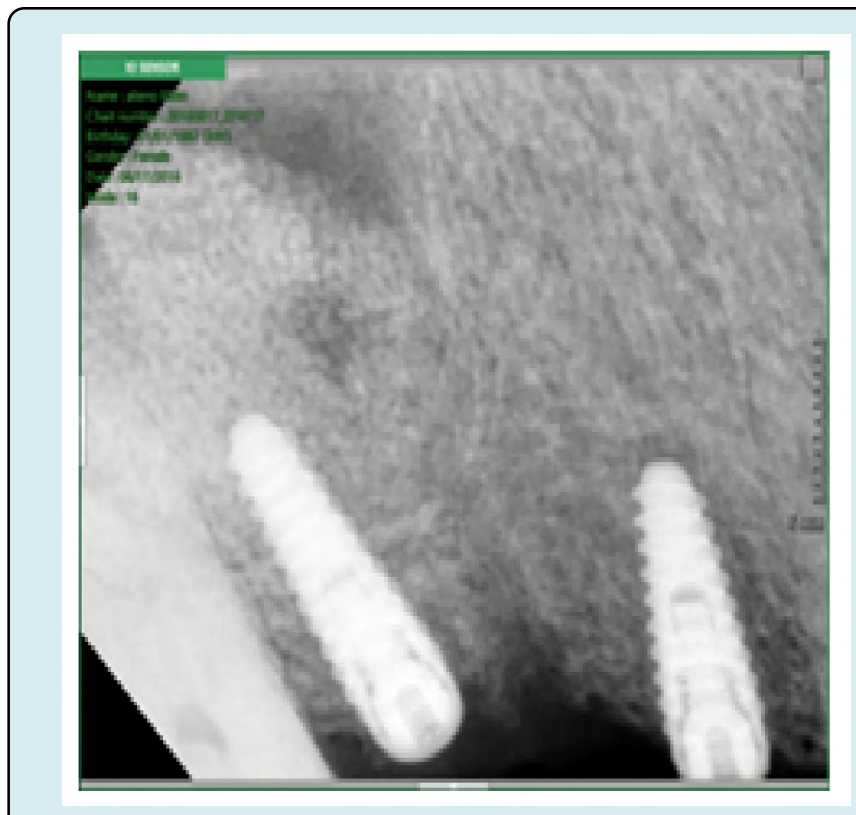

Figure 4: Patient 2 immediate post op x-ray.

Table 1: Sociodemographic and clinical characteristics of two patients were as follows -

\begin{tabular}{|c|c|c|}
\hline & Patient 1 & Patient 2 \\
\hline Age /sex & $54 /$ Female & Married \\
\hline Marital status & Married & Undergraduate \\
\hline Education level & Master degree & Christen \\
\hline Religion & Christen & Teacher \\
\hline Occupation & Accountant & No Para functional habits \\
\hline Oral habits & No Para functional habits \\
\hline Missing teeth & 16,15 & Average \\
\hline Oral hygiene & Average & Diabetic \\
\hline Medical condition & Diabetic & 4mm \\
\hline Available bone height & $3 m m$ & Periapical abscess \\
\hline Cause of tooth loss & Periodontitis & \\
\hline
\end{tabular}

\section{Discussion}

Availability of inadequate bone in the posterior edentulous maxilla is always a limitation for the implant placement. To overcome this problem sinus lift and floor augmentation has become a routine procedure. There are two types of technique for sinus lift, direct sinus lift and indirect sinus lift. Selection of technique usually depends on the available bone height below sinus floor. Direct sinus lift originally described by Tatum is usually indicated when available bone height is less than $5 \mathrm{~mm}$. Indirect sinus lift or Summers osteotomy was developed by Summer. He used osteotomes of different sizes to lift the sinus via crestal approach. This technique is indicated when available bone height is more than $6 \mathrm{~mm}$ [6]. There are various materials available as substitute of bone for grafting. Autogenously bone grafts are always considers as the best option because of its osteoconductive and osteoinductive potential [8]. But according to the literature implants placed in grafts which mixture is of autogenously and synthetic material have better outcome than implants using only Autogenously grafts [9]. Autogenously bone grafts even though they are best options but they need second surgical site which is not comfortable with many patients. To avoid second surgical site other type of grafting materials like allografts and xenografts are also very popular. Now a days sinus lift surgery became very popular for maxillary posterior implants. This surgery is related with many complications also. Considering the complications, 


\section{International Journal of Surgery \& Surgical Techniques}

sinus membrane perforation was found to be the most common complication. Sinus perforation does not affect the ossteointegration of implants if the size of perforation is less than $5 \mathrm{~mm}$. if the size of perforation is larger than $5 \mathrm{~mm}$ it can be repaired and results showed that repaired sinus with graft and ePTFE membranes also have good prognosis [10-12]. In conclusion, sinus lift surgery followed by augmentation of floor and immediate implant placement is very good option for restoring edentulous atrophic maxilla provided proper planning, case selection and aseptic technique has been followed.

\section{References}

1. Daniel D, Rao SG (2012) Evaluation of increase in bone height following maxillary sinus augmentation using direct and indirect technique. J Dent Implants 2(1): 2631.

2. Chaurasia BD (2010) Human Anatomy Head \& Neck, Brain ( $3^{\text {rd }}$ edn), CBS Publishers, 3: 230-231.

3. Tantum H jr (1986) Maxillary and sinus implant reconstructions. Dent clin north AM 30(2): 207-229.

4. Smiler LB, Jensen OT (1992) Sinus lift grafts and endosseous implants treatment of the atropic posterior maxilla. Dent clin North Am 36(1): 151-158.

5. Boyne PJ, James RA (1980) Grafting of the maxillary sinus floor with autogenous marrow and bone. J Oral Surg, 38(8): 613-616.
6. Metodi A (2009) Alternative Sinus Lift Techniques Literature review. Journal of IMAB - Annual Proceeding (Scientific Papers) 2: 23-27.

7. Javed F, Vohra F, Zafar S, Almas K (2014) Significance of osteogenic surface coatings on implants to enhance osseointegration under osteoporotic-like conditions. Implant Dent 23(6): 679-686.

8. Wallace SS, Froum SJ (2003) Effect of maxillary sinus augmentation on the survival of endosseous implants. A systematic review. Ann Periodontol 8(1): 328-343.

9. Smiler DG, Johnson PW, Lozada JL, Misch C, Rosenlicht JL, et al. (1992) Sinus lift grafts and endosseous implantsTreatment of the atrophic posterior maxilla. Dent Clin North Am 36(1): 151-188.

10. Schwartz-Arad D, Herzberg R, Dolev E (2004) the prevalence of surgical complications of the sinus graft procedure and their impact on implant survival. J Periodontol 75(4): 511-516.

11. Barone A, Santini S, Sbordone L, Crespi R, Covani U (2006) A clinical study of the outcomes and complications associated with maxillary sinus augmentation. Int J Oral Maxillofac Implants 21(1): 81-85.

12. Pal U, Sharma NK, Singh R, Mahammad S, Mehrotra D, et al. (2012) Direct vs. indirect sinus lift procedure: A comparison. Nat j maxillofac surg 3(1): 31-37. 